\title{
Estratégias de controle do medo e ansiedade em pacientes odontopediátricos: revisão de literatura
}

\author{
Strategies for the control of fear and anxiety in odontopediatric patients: a literature review \\ Estrategias para el control del miedo y la ansiedad en pacientes odontopediátricos: una \\ revisión de la literatura
}

Maria Eduarda Brandão Balbino Torres ${ }^{1 *}$, Karina Livia Barros Souza1, Victor Santos Andrade Cruz ${ }^{1}$.

\section{RESUMO}

\begin{abstract}
Objetivo: Verificar os fatores relacionados à ansiedade infantil e o atendimento odontológico e as estratégias adotadas para seu controle por meio de análises bibliográficas. Métodos: Foi realizado uma revisão de literatura, com abordagens informativas, do qual o processo de formulação baseou-se por intermédio de pesquisas em artigos científicos, em bases de dados, possuindo como seus respectivos descritores: Ansiedade ao Tratamento Odontológico, Odontopediatria, Reabilitação Bucal. Resultados: A princípio foi analisado o desencadeamento da ansiedade associada ao medo da criança em ir ao Cirurgião Dentista (CD), desse modo, podendo prejudicar a interrelação entre o profissional e o paciente. O CD necessita dialogar com os responsáveis sobre técnicas de manejo comportamental que são usadas nos procedimentos odontopediátricos, técnicas essas que proporcionam o tratamento a criança com confiança e convicção no trabalho do profissional. Considerações finais: No assistencialismo o entendimento da ansiedade infantil é fundamental para manejar corretamente a consulta e reduzir à sensação de medo no paciente, assim sendo, o estudo mostra estratégias de manejo comportamental pediátrico e sua importância, modificando o atendimento clínico odontopediátrico para menos inquietante e mais eficaz.
\end{abstract}

Palavras-chave: Ansiedade ao tratamento odontológico, Odontopediatria, Reabilitação bucal.

\section{ABSTRACT}

Objective: To verify the factors related to childhood anxiety and dental care and the strategies adopted for its control through bibliographic analyzes. Methods: A literature review was carried out, with informative approaches, of which the formulation process was based through research in scientific articles, in databases, having as their respective descriptors: Anxiety to Dental Treatment, Pediatric Dentistry, Oral Rehabilitation. Results: At first, the triggering of the anxiety associated with the child's fear of going to the Dental Professionals was analyzed, thus, it could harm the interrelation between the professional and the patient. The dentist needs to dialogue with those responsible about behavioral management techniques that are used in pediatric procedures, techniques that provide treatment to the child with confidence and conviction in the professional's work. Final considerations: In assistentialism, the understanding of childhood anxiety is essential to correctly manage the consultation and reduce the feeling of fear in the patient, therefore, the study shows pediatric behavioral management strategies and their importance, changing the pediatric clinical care to less disturbing and more effective.

Keywords: Dental anxiety, Pediatric dentistry, Mouth rehabilitation.

\section{RESUMEN}

Objetivo: Verificar los factores relacionados con la ansiedad infantil y el cuidado dental y las estrategias adoptadas para su control mediante análisis bibliográficos. Métodos: Se realizó una revisión de la literatura, con enfoques informativos, cuyo proceso de formulación se basó en la investigación en artículos científicos, en bases de datos, teniendo como sus respectivos descriptores: Ansiedad al Tratamiento Dental, Odontopediatría, Rehabilitación Oral. Resultados: En un primer momento se analizó el desencadenamiento de la ansiedad asociada al miedo del niño a acudir al Cirujano Dentista, por lo que podría perjudicar la interrelación entre el profesional y el paciente. El odontólogo necesita dialogar con los responsables sobre las técnicas de manejo conductual que se utilizan en los procedimientos pediátricos, técnicas que brinden tratamiento al niño con confianza y convicción en el trabajo del profesional. Consideraciones finales: En asistencialismo, la comprensión de la ansiedad infantil es fundamental para manejar correctamente la consulta y disminuir la sensación de miedo en el paciente, por ello, el estudio muestra las estrategias de manejo conductual pediátrico y su importancia, cambiando la atención clínica pediátrica a menos perturbadora y más efectiva.

Palabras clave: Ansiedad al tratamiento odontológico, Odontología pediátrica, Rehabilitación bucal.

${ }^{1}$ Centro Universitário CESMAC (CESMAC), Maceió - AL. *E-mail: mariaeduardabbt@outlook.com 


\section{INTRODUÇÃO}

O atendimento odontopediátrico é ligado diretamente ao profissional em compreender os quesitos emocionais infantis (OLIVEIRA MF, et al., 2012). A ansiedade odontológica refere-se a uma reação emotiva definida por sensações de aflição, angústia, tensão e inquietude. Na situação de intimidação, não existe um agente concreto, quão maior a ansiedade da criança, maior será sua susceptibilidade à dor. A criança ansiosa no consultório odontológico é resultado da ansiedade materna e consequentemente o comportamento infantil negativo no consultório (MEDEIROS LA, 2013).

A contextualização da psicologia moderna explica que as manifestações internas dos comportamentos desempenham influências na percepção do ambiente odontológico, de forma que proporcionam uma expectativa maior da dor (COSTA RSM, et al., 2012). Isto é, quão mais ansiosa a pessoa estiver, mais alta possibilidade de respostas de repulsa aos procedimentos odontológicos. A ausência do entendimento ao processo que será feito no consultório, pode refletir numa maior possibilidade de reações de tensão e ansiedade. Segundo as teorias apresentadas anteriormente, as manifestações das respostas emocionais podem ser explicadas como ameaças pelos pacientes que farão procedimentos odontológicos (ZANATTA J, et al., 2014).

A visão obtida nos dias de hoje ao que se refere a odontologia é interligado diretamente as formas de medos que os pacientes possuem, principalmente os que apresentam um quadro de ansiedade, o que demanda um ponto importante: coordenar essa adversidade em prol de uma boa conexão entre o profissional, o paciente e seus responsáveis (HASS MGM, et al., 2016). Desse modo, é fundamental entender que um bom tratamento não é suficiente, o atendimento infantil se tornou uma característica diferencial entre cirurgiões-dentistas que conseguem conquistar a confiança das crianças e são capazes de efetuar seu trabalho sem adversidades comportamentais (FERREIRA R, et al., 2016).

Compreendido essa característica, o profissional gera competência para enfrentar os pacientes diferenciados na consulta odontológica. Então, é fundamental o entendimento da psicologia e/ou psicopatologia no consultório odontológico, com propósito de aprimorar a conexão entre o profissional e o paciente (MOURA BF, et al., 2015). Simplificando, há dois grupos das técnicas de manejo: técnicas aversivas (contenção física e mão sobre a boca) e não aversivas (falar-mostrar-fazer, reforço positivo, dessensibilização, imitação ou modelagem e distração). Também há a sedação, uso de medicamentos ansiolíticos antes ao atendimento e anestesia geral (MEJíA RC, et al., 2015).

As técnicas de manejo aversivas normalmente são menos utilizadas no manejo do paciente infantil. Porém, as crianças não colaborativas, ou, de difícil comportamento, são mais trabalhosas frente ao atendimento. Elas exigem grande atenção e cuidado para que o tratamento seja feito, porque, muitas vezes, têm medos prévios e dificuldade de compreensão da necessidade dos procedimentos (GUPTA N, et al., 2017). Assim sendo, esta revisão de literatura possui como principal objetivo distinguir os fatores relacionados à ansiedade infantil e a forma do atendimento odontológico além de demonstrar as estratégias adotadas para seu controle do medo, ocasionado por inúmeros fatores.

\section{MÉTODOS}

Relaciona-se a uma busca bibliográfica estabelecida por intermédio de uma revisão de literatura dos quais apresenta uma interpelação de forma descritiva, com caraterísticas informativas estabelecida entre os intervalos de janeiro de 2020 a agosto de 2020, cujo parâmetro foi feito através da análise de artigos publicados em bases de dados eletrônicos, Literatura Latino Americana e do Caribe em Ciências da Saúde (LILACS), Literatura Internacional em Ciência da Saúde (MEDLINE) e Scientific Electronic Library Online (SCIELO).

Para esse fim, o advento desse estudo foi determinado através dos subsequentes métodos: Emissões de publicações do Ministério da Saúde e Organizações Internacionais, no idioma inglês, espanhol e português, priorizando por artigos científicos publicados nos últimos 10 anos (2010 a 2020). Em consentimento com o seu processo de caracterização formulativa organizacional, a criação desse artigo foi procedentes através de 
3 pesquisadores: inicialmente foi estabelecido a temática a ser estudada, investigando e sucessivamente, foram estabelecidas as leituras devidas de maneira aprofundada para a aplicação dos processos de maneira descritiva, deixando-os assim mais restringidos.

Após essa leitura foram estabelecidos os artigos que iriam ser englobados, com o propósito de examinar os artigos que se referia a presente revisão. Com o estabelecimento definido, foram interligados as partes das centrais que estabeleciam as solicitações, como a grandeza das amostragens, o objetivo geral e especifico, seus métodos empregados e os principais resultados, que se exibiram as estratégias de controle do medo e ansiedade em pacientes odontopediátricos, tendo como fundamentais metodologias de restrições os períodos de publicações, trabalhos de ensaios que não possuíam controle e os artigos que não possuíam relevância com a temática e não estavam presentes na integra, nos artigos selecionados foram observados os aspectos que levam a uma criança a possuir medo ou ansiedade no consultório odontológico. Os descritores utilizados foram: Ansiedade ao tratamento odontológico, Odontopediatria, Reabilitação Bucal.

\section{RESULTADOS E DISCUSSÃO}

Foram estabelecidos 25 artigos científicos que garantia a efetividade e integridade nas bases de dados mencionadas. Ao desenvolvimento da pesquisa, 12 possuíam várias repetições em diversas fontes de fundamento para à pesquisa, desfrutando dessa maneira de 13 artigos, porém 05 foram abortados por não possuir de forma objetiva com as centrais de diretrizes do estudo. Em consequência desses fatores 08 artigos foram examinados para o processo avaliativo por meio de sínteses que estavam presentes, assim sendo, nenhum outro artigo foi excluído, desta maneira, instituiu-se 08 artigos para a elucidação e estabelecimentos indispensáveis para o aprimoramento do estudo.

Posteriormente, após o processo organizacional das conjunturas do presente estudo, não foram sobrepostas outras fontes de pesquisa, a revisão foi ajustada por 08 artigos científicos relevantes, e 0 fluxograma da Figura 1 assegura as formulações de pesquisas que foram estabelecidas em concordância com a otimização efetivada.

Figura 1 - Fluxograma dos estudos identificados.
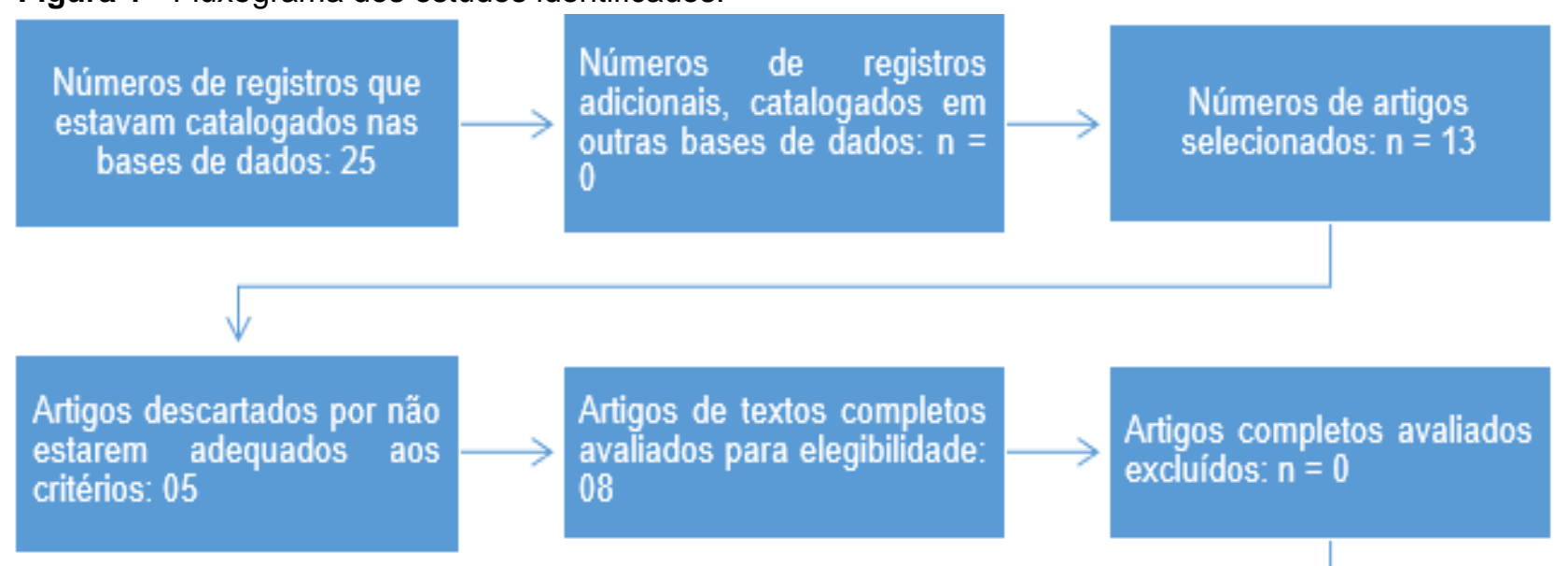

Artigos completos avaliados excluídos: $n=0$

Artigos de textos completos

selecionados: $n=08$

Fonte: Torres MEBB, et al., 2020.

Os respectivos artigos dispuseram suas principais informações através dos tópicos: artigo/autor, ano, periódico, objetivo e resultados, dos quais foram tabulados e expostos na Quadro 1. 
Quadro 1 - Delineamento, métodos e principais desfechos dos estudos selecionados.

\begin{tabular}{|c|c|c|c|c|}
\hline Artigo/Autor & Ano & Periódico & $\begin{array}{c}\text { Objetivo } \\
\end{array}$ & $\begin{array}{l}\text { Resultados } \\
\end{array}$ \\
\hline $\begin{array}{l}\text { (JAFARZADEH } \\
\text { M, et al.) }\end{array}$ & 2013 & Adv. Biomed. & $\begin{array}{l}\text { Investigar o efeito da aromaterapia com óleo essencial de } \\
\text { laranja na ansiedade infantil durante o tratamento } \\
\text { odontológico. }\end{array}$ & $\begin{array}{l}\text { Cada criança foi submetida a duas consultas de tratamento odontológico, } \\
\text { incluindo dentário profilaxia e terapia selante de fissuras sob aroma de } \\
\text { laranja em uma sessão (intervenção) e sem aroma (controle) em outra. }\end{array}$ \\
\hline (AGARWAL M.) & 2013 & $\begin{array}{l}\text { J Indian Soc } \\
\text { Pedod Prev } \\
\text { Dent }\end{array}$ & $\begin{array}{l}\text { Analisa através de um estudo transversal, Venham Picture, } \\
\text { um teste psicométrico, foi usado para estimar a prevalência } \\
\text { de ansiedade odontológica em crianças na faixa etária de } 6 \\
\text { a } 10 \text { anos, com e sem experiência odontológica anterior. }\end{array}$ & $\begin{array}{l}\text { O reconhecimento apropriado da ansiedade do paciente pode solidificar } \\
\text { sua confiança e permitir ao dentista pediátrico uma revisão informada } \\
\text { das opções de tratamento potenciais específicas para cada indivíduo. }\end{array}$ \\
\hline (KAUR R, et al.) & 2015 & $\begin{array}{l}\mathrm{J} \text { Indian } \\
\text { SocPedodPrevD } \\
\text { ent }\end{array}$ & $\begin{array}{l}\text { Avaliar e comparar auxílios de distração áudio e audiovisual } \\
\text { no tratamento de pacientes odontológicos pediátricos } \\
\text { ansiosos de diferentes faixas etárias e estudar a resposta } \\
\text { das crianças a consultas odontológicas sequenciais com o } \\
\text { uso de auxílios de distração. }\end{array}$ & $\begin{array}{l}\text { O teste post-hoc de diferença significativa honesta de Tukey com nível } \\
\text { de significância de } 0,05 \% \text { revelou que o grupo audiovisual apresentou } \\
\text { diferença estatisticamente altamente significativa do grupo de áudio e } \\
\text { controle. }\end{array}$ \\
\hline $\begin{array}{l}\text { (MATOS CV, et } \\
\text { al.) }\end{array}$ & 2015 & $\begin{array}{l}\text { Rev Gestão \& } \\
\text { Saúde }\end{array}$ & $\begin{array}{l}\text { Analisar as práticas de promoção em saúde bucal relatadas } \\
\text { na literatura através de uma revisão integrativa na base de } \\
\text { pesquisas da Bireme. Identificaram-se três tendências nos } \\
\text { programas analisados, e em sua maioria apontaram para a } \\
\text { necessidade de se repensar as práticas educativas. }\end{array}$ & $\begin{array}{l}\text { Embora a odontologia se mostre muito desenvolvida em tecnologia, não } \\
\text { responde em níveis significativos às demandas dos problemas de saúde } \\
\text { bucal da população. Nesse contexto, a educação em saúde bucal tem } \\
\text { sido cada vez mais requisitada, considerando o baixo custo e as } \\
\text { possibilidades de impacto odontológico no âmbito público e coletivo. }\end{array}$ \\
\hline $\begin{array}{l}\text { (MCCONNELL T, } \\
\quad \text { et al.) }\end{array}$ & 2016 & $\begin{array}{l}\text { Scientifi c } \\
\text { Electroni c } \\
\text { Library } \\
\text { (SCIELO) }\end{array}$ & $\begin{array}{l}\text { Garantir uma base metodológica sólida para o } \\
\text { desenvolvimento de um ensaio robusto randomizado de fase } \\
\text { III de musicoterapia para melhorar a qualidade de vida em } \\
\text { pacientes em cuidados paliativos. }\end{array}$ & $\begin{array}{l}\text { Este estudo piloto e de viabilidade testou procedimentos, resultado e } \\
\text { ferramenta validadas; estimando as taxas de recrutamento e evasão; e } \\
\text { calculou o tamanho da amostra necessária para um ensaio randomizado } \\
\text { de fase III para validação da eficácia da musicoterapia na melhoria da } \\
\text { qualidade de vida. }\end{array}$ \\
\hline $\begin{array}{l}\text { (MENDONÇA } \\
\text { JGA, et al.) }\end{array}$ & 2016 & Rev. Odontol. & $\begin{array}{l}\text { Avaliar a eficácia e eficácia relativa dos agentes de sedação } \\
\text { consciente e dosagens para o controle do comportamento } \\
\text { em odontopediatria. }\end{array}$ & $\begin{array}{l}\text { Há algumas evidências de certeza moderada de que o midazolam oral é } \\
\text { um agente sedativo eficaz para crianças em tratamento odontológico. Há } \\
\text { necessidade de outros ensaios clínicos bem planejados e relatados para } \\
\text { avaliar outros agentes potenciais de sedação. }\end{array}$ \\
\hline $\begin{array}{l}\text { (SILVA LFP, et } \\
\text { al.) }\end{array}$ & 2016 & Rev. Odontol. & $\begin{array}{l}\text { Revisar e discutir por meio de literaturas as técnicas de } \\
\text { controle comportamental em odontopediatria. }\end{array}$ & $\begin{array}{l}\text { Para que tais técnicas sejam mais efetivas, é necessário conhecer suas } \\
\text { possíveis restrições quanto à faixa etária e perfil de cada criança, de } \\
\text { modo a promover e estabelecer segurança e qualidade ao atendimento. }\end{array}$ \\
\hline $\begin{array}{l}\text { (SHAHNAVAZ S, } \\
\quad \text { et al.) }\end{array}$ & 2018 & $\begin{array}{l}\text { Journal of } \\
\text { Medical Internet } \\
\text { Research }\end{array}$ & $\begin{array}{l}\text { Testar a hipótese de que o ICBT (Terapia cognitvo- } \\
\text { comportamental na internet). guiado por psicólogo melhora a } \\
\text { capacidade de crianças e adolescentes em idade escolar de } \\
\text { controlar a ansiedade odontológica por (1) diminuir a } \\
\text { evitação e afetar o diagnóstico de fobia e (2) diminuir o medo } \\
\text { dentário e aumentando a autoeficácia dos grupos-alvo. O } \\
\text { estudo também teve como objetivo examinar a viabilidade e } \\
\text { aceitabilidade deste novo tratamento. }\end{array}$ & $\begin{array}{l}\text { ICBT é um tratamento promissor e viável para ansiedade odontológica } \\
\text { em crianças e adolescentes. Integrá-lo ao atendimento odontológico } \\
\text { pediátrico de rotina aumentaria o acesso a um tratamento psicológico } \\
\text { eficaz. }\end{array}$ \\
\hline
\end{tabular}

Fonte: Torres MEBB, et al., 2020. 
A odontologia para pacientes infantis é uma especialidade que demanda do profissional a ser capaz de efetuar prevenção e ter capacidade técnica para a realização do tratamento odontológico infantil ou no adolescente, assim como possuir uma atenção especial com o seu bem-estar durante a efetivação dos procedimentos (CADERMATORI MG, 2014).

A fobia relacionada ao tratamento dentário em pacientes infantis pode causar disfunções graves de saúde e perdurar até a juventude se tornando um comportamento problemático no decorrer da consulta odontológica. Faz-se de extrema importância compreender esses pacientes ansiosos antecipadamente prevenindo essa adversidade comportamental (KHANDURI N, et al., 2019).

A ansiedade é uma situação emotiva determinada como sentimento não específico de apreensão, desconforto ou medo, com etiologia desconhecida (AGARWAL M, 2013). Quando associada aos procedimentos odontológicos, é a sensação causada por eventos ligados ao atendimento que ocasionam preocupação e incômodo, gerando perspectiva negativa. Esta circunstância emocional pode incluir fatores fisiológicos, mentais, emotivos e comportamentais (ARMFIELD JM e HEATON LJ, 2013).

Ranali J (2015) confirma que a manifestação do medo é ligada aos cinco medos básicos do ser humano: do desamparo, do desconhecido, da dor, da dependência, da mutilação, da mudança, do corpo e da morte. É constatado que a ansiedade e o medo ligadas às vivências odontológicas dos pacientes, são considerados barreiras pertinentes no atendimento (CREGO A, et al., 2014).

É uma sensação importante dentro da odontologia infantil, visto que é capaz de provocar divergentes tipos de atitudes na criança, que influenciam e complicam o atendimento odontopediátrico como choro, pirraça, esquiva ao tratamento e mudanças fisiológicas, tais como, aumento da frequência cardíaca e da saturação de oxigênio. $O$ trabalho da odontopediatra no atendimento da criança ansiosa é compreender esta adversidade, fazendo o uso técnicas de manejo do comportamento (PORRITT J, et al., 2013).

A ideia de dor agregada ao tratamento odontológico é antecessora de medo e ansiedade há tempos e o rótulo negativo do cirurgião-dentista é visto frequentemente em todo o mundo (MARTINS RJ, et al., 2017). Mesmo com os progressos tecnológicos na odontologia no decorrer dos anos, sensações negativas continuam diariamente, vividos nesta especialidade (SHAHNAVAZ S, et al., 2018). As causas mais relevantes para a fobia e ansiedade odontopediátrica são comportamentos e experiências negativas maternas e seus palpites sobre procedimentos odontológicos (KAUR R, et al., 2015).

Uma das concepções fundamentais ligadas às técnicas de comportamento é que os responsáveis necessitam de esclarecimento e consciência do que pode ser feito se a criança não apresentar um comportamento colaborativo. Visto que provavelmente os pais vão influenciar de forma direta na autorização da técnica proposta pelo profissional, uma vez que esse contexto gera uma ansiedade também aos responsáveis do paciente (SILVA LFP, et al., 2016).

Alguns estudos investigaram a ansiedade e as atitudes das crianças durante os atendimentos e analisado simultaneamente a ansiedade da mãe com o objetivo de descobrir ligações entre o estado emocional das duas. Percebeu-se que a ligação mais clara da ansiedade materna e colaboração infantil no ambiente odontológico ocorrem constantemente em menores de cinco anos, porque, de acordo com hipóteses psicológicas do desenvolvimento, são mais ligados a suas mães.

Nesses estudos, percebeu-se a influência da ansiedade da mãe sobre o grau de desenvolvimento da ansiedade do filho. Também analisaram que a ansiedade odontológica tem conexão com o procedimento que será feito e deduziram que grande parte das crianças e mães manifesta ansiedade dental leve. Diferente do esperado, mães com alta ansiedade dental não possuem filhos com alta ansiedade dental (MENDONÇA JGA, et al., 2016).

A ansiedade materna reflete nas atitudes dos filhos e na consulta com a odontopediatra, principalmente dos três aos sete anos de idade. Além disso, há relatos de associação entre o tempo tardio de amamentação e a insegurança da criança em lidar com situações novas, porque este laço simbiótico materno dificulta a criança de desprender-se da figura da mãe (JAFARZADEH M, et al., 2013). 
Matos CV, et al. (2015), afirma que é imprescindível instruir corretamente a família da criança, de forma que não seja transmitida ansiedades ao paciente infantil. Silva LFP, et al (2016), argumentam que o Cirurgião Dentista necessita dialogar com os responsáveis sobre técnicas de manejo comportamental que são usadas nos procedimentos Odontopediátricos, técnicas essas que proporcionam o tratamento a criança com confiança e convicção no trabalho do profissional. Logo, o manejo comportamental viabiliza minimizar possíveis perigos de acidentes no decorrer do atendimento assegurando a efetividade do serviço. As técnicas são: de comunicação verbal, comunicação não verbal, dizer-mostrar-fazer, controle de voz, distração, reforço positivo e modelo.

O controle de voz é o uso de uma modificação proposital no tom, volume ou ritmo da voz para induzir e manipular o comportamento da criança. Essa técnica tem a intenção de chamar a atenção e a cooperação do paciente, diminuir a possibilidade de comportamentos não cooperativos e afirmar as funções esperadas do profissional e do paciente (MATOS CV, et al., 2015)

Nos casos dos pacientes extremamente ansiosos aconselha-se primeiramente escolher as técnicas de condicionamento e desempenhar abordagem psicológica. Se for urgente é indicada a terapêutica farmacológica, que usa medicações ansiolíticas, como os benzodiazepínicos ou a sedação com óxido nitroso, para estabilizar o quadro e efetuar o procedimento necessário (GIORGI et al. 2010).

É normal começar o tratamento sem procedimentos dolorosos, adiando o uso da anestesia para quando houver confiança da criança. Sabe-se que a criança nota que o cirurgião-dentista não está mentindo e assim começa a confiar, por isso o valor da técnica "dizer-mostrar-fazer", a odontopediatra deve esclarecer os procedimentos de forma clara e lúdica (MCCONNELL T, et al., 2016). Quando o paciente se sente confortável ao âmbito do consultório e da equipe de trabalho após consultas contínuas, aumenta-se a conexão e a criança inclina-se para um comportamento mais cooperativo.

Uma opção de manejo comportamental num pequeno espaço de tempo é o método de incentivação, que junto do reforço positivo, encoraja a criança na mudança de comportamento para haver uma gratificação. Há também a relevância de fazer um condicionamento remoto, observando a criança antes que ele chegue à cadeira. Tem que haver um diálogo com a criança na passagem da sala de espera para o consultório e nesse tempo o condicionamento da criança na cadeira odontológica (VASCONCELLOS C, et al., 2017).

O reforço positivo é usado para aumentar a chance de ocorrer futuramente um comportamento apropriado através de uma demonstração de um resultado positivo (chamada reforçadora) logo depois sua ocorrência. Quando elogiar deve-se sempre descrever e enaltecer de modo direto os comportamentos colaborativos. A intenção destas técnicas é melhorar o comportamento desejado, transformando-o cada vez mais frequente (JAFARZADEH M, et al., 2013).

A consulta infantil com a execução de técnicas de manejo do comportamento embasadas em conhecimentos oriundos da psicologia torna-se mais agradável. Entretanto, a sedação consciente é conveniente em alguns casos, sem substituir as técnicas mencionadas. A criança com medo e ansiedade elevados enfrentará stress fisiológico e psicológico que com a ajuda da sedação é reduzido. (MATOS CV, et al., 2015). Fármacos distintos são usados para a sedação consciente. Por exemplo: mais comuns como óxido nitroso e as benzodiazepinas, outros agentes como, hidrato cloral, cetamina, propofol, hidroxizina e opióides. (VASCONCELLOS C, et al., 2017).

Mendonça JGA, et al. (2016) analisaram a relação entre as condições pré operatórias e a dor no decorrer do procedimento odontológico sob sedação. O estudo foi feito com 27 crianças que necessitavam de tratamento restaurador e foram submetidas a sedação oral (Midazolam, Dormire $®$ ). Informações sobre idade, gênero e experiências anteriores com tratamentos dentários foram perguntadas aos pais. A dor durante 0 tratamento foi avaliada por vídeos feitos durante o tratamento odontológico.

A maior parte das crianças $51 \%$ não apresentou dor durante o tratamento sob sedação, e foram observados escores de dor baixos ou moderados. A dor durante o procedimento odontológico sob sedação foi pouco observada neste grupo, de baixa intensidade e não se associou a idade, gênero, condição bucal e experiência odontológica anterior. 
Possibilidades menos invasivas que a sedação farmacológica apresentam repercussões muito satisfatórias na diminuição da ansiedade no atendimento odontopediátrico. A organização do âmbito do consultório por meio da exclusão de objetos e sons que despertam ansiedade e medo, o uso de essências de óleos essenciais no local de espera, utilização de óculos de realidade virtual durante os procedimentos mais invasivos (KAUR R, et al., 2015).

O método de distração é uma das técnicas orientadas pela literatura a ser usada no manejo do paciente infantil durante a consulta odontológica. Esta estratégia compreende em afastar a atenção da criança de sua concepção de procedimentos vistos como indelicados, é capaz ser feita de modo ativo ou passivo (NUVVULA S, et al., 2015). Na utilização da técnica distração do paciente pode-se utilizar músicas, vídeos, histórias infantis e outros recursos audiovisuais através de TVs e tablets. A música, além de relaxar a criança, se sobrepõe ao som dos aparelhos odontológicos. Interagir com temas diferentes e permitir que a criança segure algum brinquedo são técnicas que agregam e cooperam para um bom relacionamento da criança com o profissional (SILVA LFP, et al., 2015).

A música é utilizada em consultórios pelos profissionais como uma tática no atendimento para aprimorar o bem-estar dos pacientes (UGGLA, L, et al.,2018). Ela poderá ser utilizada como uma alternativa na redução da ansiedade e possibilitar um ambiente confortável e relaxante para a criança, assim, diminuindo os níveis de cortisol (MENDONÇA JGA, et al., 2016).

Mendonça JGA, et al. (2016), analisaram se existia mudanças no comportamento das crianças após receberem imagens neutras e imagens positivas antes da consulta e não ocorreu distinção em relação à melhora ou piora de comportamento entre os grupos que receberam imagens diferentes, mas as neutras como as positivas possuem o impacto positivo igual, aperfeiçoando o comportamento infantil durante 0 tratamento odontológico. A finalidade dessa técnica é dar uma antevisão para o paciente do que esperar no decorrer do seu atendimento e elaborar um ambiente de questionamento e curiosidade com o consultório odontológico, que devem ser respondidos pela odontopediatra anteriormente ao atendimento (SHAHNAVAZ S, et al., 2018).

A técnica de mão-sobre-a-boca é usada com a intenção de facilitar o tratamento odontológico em crianças que não possuem de necessidades especiais, que demonstrem comportamentos difíceis, barulhentos, extremamente não colaborativos e que dificultam na realização do procedimento. Se a criança continue sendo não cooperativa, o profissional deverá utilizar a variação da técnica, somente com autorização dos responsáveis, que é a mão sobre a boca com restrição das vias aéreas. Mesmo sendo autorizada pela American Academy of Pediatric Dentistry (AAPD), a técnica mão-sobre-a-boca continua a mais polêmica entre as técnicas usadas por odontopediatras (SILVA LFP, et al., 2016).

A utilização da contenção física é feita em crianças não cooperativas em que não seguem às técnicas de manejo comportamental e condicionamento passivo, complicando o tratamento odontológico (CADERMATORI MG, 2014). Continuando o pensamento de acalmar a atitude das crianças no decorrer do atendimento infantil, Matos CV, et al. (2015) analisaram os métodos de distração audiovisuais durante o procedimento odontológico e concluíram que eles permitem a consulta seja mais tranquilo com pacientes ansiosos. Segundo seus estudos, os métodos audiovisuais são superiores a somente os auditivos, mas ambos são julgados uma boa escolha no manejo de pacientes ansiosos.

$O$ atendimento infantil humanizado é obrigado para reconhecer o grau de ansiedade da criança e pelo uso de técnicas menos invasivas na aproximação destes pacientes, que escolham métodos que não utilizem medicações ou qualquer modo de contenção física (AGARWAL M, 2013).

Um tópico fundamental a ser colocado em pauta na execução de técnicas de manejo é a opinião dos pais em relação a estas técnicas. Pais entendidos foram mais compreensivos às técnicas do que os que não tiveram esclarecimentos. Assim sendo, há uma boa aceitação de pais, previamente esclarecidos quanto ao assunto, para o controle de voz e a contenção física. No entanto, não houve boa aceitação com as técnicas farmacológicas. Logo, os responsáveis, quando cientes de que algum procedimento é preciso para a reabilitação da saúde e bem-estar geral de seus filhos, se tornam aliados para o bom andamento do atendimento (SHAHNAVAZ S, et al., 2018). 
Kaur R, et al. (2015) apontaram que é fundamental para o êxito do atendimento que o manejo comportamental seja realizado, a não de colaboração dificulta chegar aos objetivos terapêuticos. As crianças trabalhosas demandam da odontopediatra e sua equipe certa habilidade, treinamento, dedicação, e personalidade suficiente para identificar a adversidade e a utilização efetiva de técnicas distintas de gestão de comportamento.

Diante dos os fatores externos que afetam as manifestações de ansiedade e medo observados no paciente infantil, complementando com comportamento ansioso da família, o profissional precisa aplicar as orientações, direcionando os modos dos pais para ajudar na efetividade do tratamento odontológico, sendo imprescindível existir um acordo de ideais e condutas que possibilitem que o tratamento do paciente infantil seja respeitoso e humano (SILVA LFP, et al., 2016).

A grande dificuldade da odontologia moderna é conseguir implantar técnicas educativas com a comunidade infantil, ensinando-a informações fundamentais e importantes para o desenvolvimento de saudáveis e prevenção das doenças bucais, sendo necessária a mudança de atitude relacionada a essas doenças que com frequência são vistas como inevitáveis pela população (MENDONÇA JGA, et al., 2016).

\section{CONSIDERAÇÕES FINAIS}

A ansiedade relacionada ao atendimento do paciente infantil é observada como uma limitante no dia a dia do cirurgião-dentista. A percepção que existem distintos graus de ansiedade e medo dentro do âmbito odontológico tem que estar no dia a dia clínico da odontopediatra. Desde o instante do diagnóstico do grau de ansiedade da criança, várias técnicas de controle e diminuição poderão ser usadas com o intuito que os atendimentos sejam menos desgastantes para o paciente e profissional. As técnicas mais utilizadas no manejo de comportamento que existem englobam desde a adaptação do meio de trabalho e da equipe e também técnicas farmacológicas como a sedação medicamentosa e analgesia relativa com oxigênio e óxido nitroso. Porém, o Cirurgião Dentista tem que ser capacitado para definir o tipo de método que será utilizado baseado no procedimento a ser executado, também na fase de desenvolvimento do paciente e de como ele se comporta com atenção nos quesitos de ética incluídos no uso de cada técnica e na autorização dos responsáveis.

\section{REFERÊNCIAS}

1. AGARWAL M. Dental anxiety prediction using Venham Picture test: a preliminary cross-sectional study. J Indian Soc Pedod Prev Dent. 2013:31(1);22-4.

2. ARMFIELD JM, HEATON LJ. Management of fear and anxiety in the dental clinic: a review. Aust Dent J. 2013: 58(4):390-407; 531.

3. CADERMATORI MG. Comportamento infantil durante consultas odontológicas sequenciais: influência de características clínicas, psicossociais e maternas, $2014 ; 45 \mathrm{p}$.

4. COSTA RSM, et al. Fatores determinantes de experiência dolorosa durante atendimento odontológico. Rev Dor. São Paulo. 2012; 13(4): 365-370.

5. CREGO A, et al. From Public Mental Health to Community Oral Health: The Impact of Dental Anxiety and Fear on Dental Status. Front. Public. Health. 2014; 2(16): 1-4.

6. FERREIRA R, et al. O uso da contenção física como técnica de condicionamento no atendimento odontológico de bebês: revisão de literatura. Revista Gestão \& Saúde, Paraná. 2016; 14(1): 31-36.

7. GUPTA N, et al. Evaluation of the role of the music as a nonpharmacological technique in manguptaagement of child patients, The journal of contemporary dental practice. 2017; 18(194-197).

8. HASS MGM, et al. Influência da vestimenta do cirurgião-dentista e do ambiente do consultório odontológico na ansiedade de crianças pré-escolares durante consulta odontológica: resultados de um estudo piloto. RFO, Passo Fundo. 2016; 21(2): 201-207.

9. JAFARZADEH M, et al. Effect of aromatherapy with orange essential oil on salivary cortisol and pulse rate in children during dental treatment: A randomized controlled clinical trial. Adv. Biomed. Rev., 2013; 6: 2-10.

10. KAUR R, et al. Comparativeevaluationoftheeffectivenessofaudioand audiovisual distraction aids in the management ofanxiouspediatric dental patients. J IndianSocPedodPrevDent. 2015: 33(3); 192-203.

11. KHANDURI N et al. The prevalence of dental anxiety and fear among 4-13-year-old Nepalese children. J Indian Soc Pedod Prev Dent, 2019; 37: 345-9.

12. MARTINS RJ, et al. Medo e ansiedade dos estudantes de diferentes classes sociais ao tratamento odontológico. Arch Health Invest, São Paulo, 2017; 6(1): 43- 47. 
13. MATOS CV, et al. A educação em saúde bucal e suas representações na atenção primária à saúde. Rev Gestão \& Saúde. São Paulo. 2015; 6, Supl. 1; 845-855.

14. MCCONNELL T, et al. Evaluation of the effectiveness of music therapy in improving the quality of life of palliative care patients: a randomised controlled pilot and feasibility study. Pilot and Feasibility Studies,2016; 70(2): 1-8.

15. MEDEIROS LA. Avaliação do grau de ansiedade dos pacientes antes de cirurgias orais menores. Revista de Odontologia da Unesp, Araçatuba. 2013; 42(5): 357-363.

16. MEJÍA RC, et al. Changes induced by music therapy to physiologic parameters in patients with dental anxiety. Complementary Therapies in Clinical Practice. 2015; 21(4): $282-286$.

17. MENDONÇA JGA, et al. Ispainduringpediatric dental sedation associate dwithchildren'spre-operative characteristics? Anexploratorystudy. Rev. Odontol. UNESP 2016; 45(5); 297-301.

18. MOURA BF, et al. Child's anxiety preceding the dental appointment: evaluation through a playful tool as a conditioning feature. RGO, Revista Gaúcha Odontologia, Campinas. 2015; 63(4): 455-460.

19. NUVVULA S, et al. Effect of audiovisual distraction with 3D video glasses on dental anxiety of children experiencing administration of local analgesia: a randomised clinical trial. Eur Arch Paediatr Dent. 2015; 16(1): 43-50.

20. OLIVEIRA MF, et al. Avaliação da Ansiedade dos Pais e Crianças frente ao Tratamento Odontológico. Pesquisa Brasileira em Odontopediatria e Clínica Integrada. 2012; 12(4): 483-489.

21. PORRITT J, et al. Assessing children's dental anxiety: a systematic review of current measures. Community Dent Oral Epidemiol. 2013; 41(2): 130-42.

22. RANALI J. Dentista pode tratar a dor causada pelo tratamento. 2015; $23 \mathrm{p}$.

23. SHAHNAVAZ S. et al. Internet-Based Cognitive Behavioral Therapy for Children and Adolescents With Dental Anxiety: Open Trial. Journal of Medical Internet Research, Estocolmo, 2018; 20(1).

24. SILVA LFP, et al. Técnicas de manejo comportamental não farmacológicas na Odontopediatria. Rev. Odontol. Univ. Cid. São Paulo, São Paulo, 2016; 28(2): 135-142.

25. SILVA LFP, et al. Técnicas de manejo comportamental não farmacológicas na Odontopediatria. Rev. Odontol. Univ. Cid. São Paulo, São Paulo. 2016; 28(2): 135-142.

26. UGGLA, L, et al. Music therapy supported the health-related quality of the life for children undergoing haematopoietic stem cell transplants, Acta paediatrica, 2018; 107(11).

27. VASCONCELLOS, C, et al. Motivation chart as a supporting tool in pediatricdentistry. RGO Ver GaúchOdontol, vol.65, n.3 Campinas, set. 2017; 16-20.

28. ZANATTA J, et al. Effects of providing prior face-to-face information on the anxiety of patients undergoing dental extraction. Revista Brasileira de Terapia Comportamental e Cognitiva, São Paulo. 2014; 17(1): 11-22. 